\title{
MIGRACIÓN INTERNACIONAL CHIAPANECA: TRAYECTORIAS DE MOVILIDAD, SOCIODEMOGRÁFICAS, Y CONDICIONES SOCIALES
}

\author{
Germán Martínez Velasco \\ El Colegio de la Frontera Sur \\ Unidad San Cristóbal \\ gmartine@ecosur.mx
}

\section{RESUMEN}

El documento refiere a la trayectoria de la evolución reciente de los destinos migratorios de la población chiapaneca, llegando a explicar los motivos de su inserción tardía al flujo hacia Estados Unidos de Norteamérica. En torno a este flujo se ofrece una caracterización sociodemográfica en términos de sus puntos de cruce, lugares de origen y de destinos, situación educativa, parentesco y sexo de la población migrante. Finalmente, el documento contribuye a esclarecer las condiciones sociales de los municipios de origen, estableciendo la asociación entre los índices de marginación, de desarrollo humano y el de intensidad migratoria, revelado éste en las categorías de municipios de alta, mediana y baja intensidad. En gran medida el documento acude a la base de información proveniente del Instituto de los Mexicanos en el Exterior, cuya exploración resulta ser una de las primeras en el tema.

Palabras clave: migración, intensidad migratoria, condiciones sociales, índice de marginación, índice de desarrollo humano. 


\title{
INTERNATIONAL MIGRATION FROM CHIAPAS: TRAJECTORIES OF MOBILITY, AND SOCIODEMOGRAPHIC AND SOCIAL CONDITIONS
}

\begin{abstract}
The document refers to the recent evolutionary trajectory of migratory destinations of the Chiapanecan population. It explains why immigrants from Chiapas inserted themselves into the immigration flow to the United States of America later than other populations. If offers a socio-demographic characterization of this migration flow in terms of crossing points, places of origin and destination, educational situation, kinship and sex of the migrant population. Finally, the document helps clarify the social conditions of the expelling municipalities, establishing an association between marginalization indexes, human development and migratory intensity in high, medium and low intensity municipalities. The document mostly resorts to the database made by the Institute for Mexicans Abroad, which is one of first themes that the article explores.
\end{abstract}

Key words: migration, migratory intensity, social conditions, marginalization index, human development index. 


\section{INTRODUCCIÓN}

Abordar el fenómeno de la migración en Chiapas nos remite a considerar sus atributos sociohistóricos referidos a sus desigualdades sociales, estructura territorial, composición étnica y orientación económica, que la han convertido en una de las entidades federativas más estudiadas de México. Las propiedades de su base social ha despertado el interés analítico desde lo histórico, antropológico, socioeconómico, político y recientemente sociodemográfico. A esa propiedad se le adicionan otra serie de atributos provenientes de su carácter fronterizo, cuya situación también le ha valido ser estudiada desde distintas aristas que van desde su conformación como frontera sur, así como de las características de sus flujos de inmigración, unas veces de carácter laboral, otras provenientes del orden político derivado de guerras intestinas de la vecina nación guatemalteca, hasta recientemente del otro gran flujo escenificado por población indocumentada. Recientemente, por la vigorosa inserción de su población en el mercado de mano de obra a Estados Unidos, también ha sido objeto de variados estudios.

En alusión a esta última temática, el presente documento establece los rasgos básicos de la emigración chiapaneca con base en una serie de indicadores provenientes de distintas fuentes de información cuantitativa que permiten plantear el estado actual de la cuestión. En la primera parte se desarrolla la trayectoria laboral de la población chiapaneca en donde se describen sus transiciones en los distintos destinos migratorios con énfasis en las características de su reciente inserción en los Estados Unidos; la segunda parte ofrece las características sociodemográficas del flujo a la Unión Americana, y en una tercera parte se presenta un análisis exploratorio entre las condiciones socioeconómicas de las regiones y municipios de origen con sus respectivos índices de intensidad migratoria a Estados Unidos. Las fuentes de información provienen básicamente de los registros del Instituto de los Mexicanos en el Exterior (IME), la Encuesta de Migración de la Frontera Norte (EMIF) y de las estadísticas del Instituto Nacional de Estadística y Geografía (INEGI), entre otras fuentes primarias y secundarias. 


\section{DE UNA MIGRACIÓN LOCAL A UNA INTERNACIONAL}

Las diversas formas de movilidad en Chiapas se han visto marcadas por tres grandes determinantes que han permitido la gestación y el desarrollo de la variedad migratoria; estas son: 1) las condiciones sociales y económicas internas; 2) su ubicación geográfica en el contexto nacional y de la región Sur-Sureste; 3) su posición fronteriza.

Respecto a la primera determinante, a manera de ilustración, resalta las condiciones históricas de desigualdad en la entidad, que propició el establecimiento, reproducción y sobrevivencia de regiones de pobreza lacerante asociadas a la población indígena asentada en la región llamada Altos, que en los primeros años del pasado siglo se constituyó en reserva de mano de obra; al tiempo que otras adquirían un dinamismo sobresaliente como la región del Soconusco, en la que se gestó y desarrolló la agricultura de plantación con su consiguiente demanda de mano de obra. Es en este marco en el que cobra vida un legendario flujo migratorio temporal de indígenas alteños a la región Soconusco, con duración de más de medio siglo sin que las condiciones sociales de desigualdad se hubiesen alterado.

En la revisión de literatura sobre este flujo laboral, en primera instancia destaca el trabajo de Pozas (1952), que describe los mecanismos premodernos de contratación de esta mano de obra como el del enganche durante varios decenios del siglo pasado, así como de otra serie de asimetrías de poder entre empleador y migrante. También el de Spenser (1988) y Martínez (1994), que describen las pugnas políticas entre indígenas chiapanecos y los grupos de poder regional, así como de la gradual incorporación de la mano de obra guatemalteca en la mencionada región. Hacia los años 80 se constata que todavía buena parte de esta población indígena se mantenía asalariada, alternando sus trabajos por cuenta propia en sus pobres tierras agrícolas con sus ingresos monetarios y lo que estos representaban en sus ingresos totales (Wasserstrom 1989). En el segundo quinquenio de los 90, en un levantamiento de campo realizado en cinco municipios indígenas de la región Altos, Martínez (1999a) describe la ausencia de estos contingentes en las plantaciones de 
café de El Soconusco y señala el peso de otros destinos intraestatales, como las plantaciones de café de la región Frailesca a algunas ciudades de la entidad, así como del traslado familiar con motivo del arrendamiento de tierras ociosas cuyos propietarios de las tierras bajas de Chiapas eran incapaces de cultivar. Ya avanzado el pasado siglo, con base en un diagnóstico general elaborado por el Consejo Estatal de Población del estado de Chiapas (COESPO 2007) se advierte que hacia los años 70 la población chiapaneca comenzaba a aparecer en otras entidades del país.

De acuerdo con el Cuadro 1, podemos apreciar que el Distrito Federal constituyó durante tres décadas $(1970,1980,1990)$ el primer destino nacional para la población chiapaneca, mientras la entidad tabasqueña ocupó ese lugar durante el decenio del año 2000. En ese mismo año, Quintana Roo aparecía en quinto lugar, también aparece en octavo lugar Baja California. Cinco años después, en 2005, estas dos últimas entidades alcanzaron los dos primeros lugares.

Cuadro 1. Principales destinos nacionales 1970, 1990, 2000 y 2005.

\begin{tabular}{|l|c|l|c|l|c|l|c|}
\hline Entidad & $\mathbf{1 9 7 0}$ & Entidad & $\mathbf{1 9 9 0}$ & Entidad & $\mathbf{2 0 0 0}$ & Entidad & $\mathbf{2 0 0 5}$ \\
\hline D. F. & 34.8 & D. F. & 17.3 & Tabasco & 14.8 & B. C. & 14.4 \\
\hline Tabasco & 19.6 & Veracruz & 16.6 & México & 14.0 & Q. Roo & 13.6 \\
\hline Veracruz & 15.6 & Tabasco & 15.7 & D. F. & 11.7 & Veracruz & 12.1 \\
\hline Oaxaca & 6.7 & México & 13.6 & Veracruz & 11.1 & México & 11.5 \\
\hline México & 6.0 & Campeche & 6.6 & Q. Roo & 8.5 & Oaxaca & 9.6 \\
\hline Campeche & 3.6 & Oaxaca & 6.5 & Campeche & 6.4 & Puebla & 4.8 \\
\hline Tamaulipas & 2.1 & Q. Roo & 4.8 & Oaxaca & 5.6 & Tabasco & 4.7 \\
\hline Guanajuato & 1.9 & Puebla & 3.4 & B. C. & 5.4 & Puebla & 3.9 \\
\hline Puebla & 1.8 & Jalisco & 2.0 & Puebla & 3.1 & & \\
\hline B. C. & 1.5 & B. C. & 1.5 & Jalisco & 2.4 & & \\
\hline
\end{tabular}

Fuente: Estimación de COESPO con base en INEGI 1970, I990, 2000, 2005; X, XI, XII Censo General de Población y Vivienda 2000, Conteo de población 2005.

En síntesis, con base en las fuentes documentales arriba descritas, en materia de migración 
interna, las grandes tendencias migratorias desarrolladas por parte de la población chiapaneca había consistido en el pasaje de una migración intraestatal de trabajo temporal asalariado en establecimientos agrícolas durante un largo periodo que va de 1920 a 1970; de arrendamiento de tierras en áreas de gran concentración terrateniente de 1940 hasta finales de 1990; del intermitente trabajo asalariado y por cuenta propia en centros urbanos iniciado en la década de 1970 a la fecha, para pasar a una migración regional y nacional significativamente a la ciudad de México, y en menor medida Tabasco, Veracruz y Estado de México de 1970 a 1990; así como los primeros visos de una migración de mayor distancia hacia los años 2000-2005 con dirección básicamente al estado de Quintana Roo, principalmente a Cancún y Playa del Carmen; y a Baja California, especialmente a Tijuana, como preámbulo de un destino internacional que sería a Estados Unidos.

\section{CHIAPANECOS EN ESTADOS UNIDOS, UNA INSERCIÓN TARDÍA PERO VIGOROSA}

En estudios recientes sobre la migración de mexicanos a Estados Unidos se ha venido señalando la incorporación de una serie de entidades migratorias llamadas emergentes en el flujo laboral a diversos Estados de la Unión Americana como elemento diferenciador de otra serie de entidades tradicionales de migración internacional. En el grupo de las primeras se encuentran los estados de Zacatecas, Durango, Jalisco, Guanajuato, Michoacán, Nayarit, San Luis Potosí, Aguascalientes y Colima; en tanto que en el de las segundas se indican a las entidades de Puebla, Estado de México, D. F., Hidalgo, Morelos, Tlaxcala, Guerrero, Oaxaca y Querétaro.

Como se sabe, estas últimas coinciden en ser las que con mayor intensidad desde decenios atrás se han incorporado masivamente al mercado laboral de Estados Unidos con una serie de impactos sociales y económicos en sus lugares de origen.

El entendimiento de este proceso diferenciador nos remite a clarificar el papel que jugaron las distintas entidades federativas de México en el Programa Bracero instrumentado entre 1942 y 1964 a iniciativa de Estados Unidos en el contexto de la Segunda Guerra Mundial. 
De la lectura sobre el tema (Durand 2007), se desprende que dicho programa fue la continuidad de otra serie de acuerdos que México y Estados Unidos habían tenido con anterioridad, sin dejar de mencionar que desde décadas atrás de por sí algunas poblaciones de la frontera ya incursionaban en el mercado laboral agrícola en áreas adyacentes a la línea fronteriza. Cabe señalar que en el citado Programa, Chiapas se ubicaba como una entidad con una participación casi nula, situación diferente a otras entidades como Guanajuato, Jalisco, Michoacán, Oaxaca o Zacatecas. Las diferencias se basan en sus porcentajes de participación provenientes de una muestra de 4 años de los 22 en los que funcionó el programa. De acuerdo con el Cuadro 2, el estado de Chiapas aparece en la lista de entidades con uno de los valores más bajos, además de que su participación solamente fue en los dos primeros años del periodo observado con $0.03 \%$ del total de braceros contratados en 1943; y $0.26 \%$ en el año 1944, valores que no llegaron tan siquiera a representar la unidad. Si se compara con Michoacán, se puede apreciar sus contrastantes diferencias en tanto que éste, en tres de los mismos cuatro años observados, su porcentaje ascendió a 26.73\% en 1943; a $4.39 \%$ en 1944 y $6.25 \%$ en 1945 . Otro estado que hasta hoy día destaca por su grado de articulación laboral con la Unión Americana es el de Oaxaca, que ya desde 1944 estaba participando con 2.04\%; en 1945 con 6.35\%, y en 1946 con 6.56\% (Durand 2007).

Cuadro 2. Braceros a Estados Unidos según porcentaje de participación por entidad federativa, 1943-1946.

\begin{tabular}{|l|r|r|r|r|}
\hline \multicolumn{1}{|c|}{ Entidad/Año } & $\mathbf{1 9 4 3}$ & \multicolumn{1}{|c|}{$\mathbf{1 9 4 4}$} & \multicolumn{1}{c|}{$\mathbf{1 9 4 5}$} & \multicolumn{1}{c|}{$\mathbf{1 9 4 6}$} \\
\hline Chiapas & 0.03 & 0.26 & 0.00 & 0.00 \\
\hline Distrito Federal & 29.18 & 66.94 & 41.21 & 0.00 \\
\hline Guanajuato & 17.09 & 4.39 & 5.38 & 0.00 \\
\hline Guerrero & 1.20 & 1.57 & 3.85 & 0.00 \\
\hline Hidalgo & 1.40 & 1.48 & 1.65 & 3.83 \\
\hline Jalisco & 5.34 & 0.00 & 0.87 & 8.70 \\
\hline Michoacán & 26.73 & 4.39 & 6.25 & 0.00 \\
\hline Oaxaca & 0.00 & 2.04 & 6.35 & 6.56 \\
\hline Veracruz & 0.00 & 2.16 & 3.93 & 0.00 \\
\hline Zacatecas & 5.86 & 1.10 & 8.28 & 37.95 \\
\hline
\end{tabular}

Fuente: Elaboración propia con base en Durand (2007). 
Sin duda que la experiencia de los estados de México en el programa bracero fue determinante en la configuración de las trayectorias migratorias de cada uno de ellos, en tanto que su participación o no devino en la conformación de una serie de conductas individuales, colectivas y comunitarias, que a manera de habitus habrían de desplegar una serie de estrategias de inserción laboral en la Unión Americana, sobre todo en lo que se refiere al desarrollo de redes sociales de apoyo mutuo, toma de decisiones, cruce e inserción en el mercado de trabajo, que a la larga redundarían en más migración.

La virtual ausencia de la entidad en el programa bracero alude a una serie de circunstancias estructurales en Chiapas que hacían difícil su participación, tales como 1) la lejanía de Chiapas y sus reservas de mano de obra respecto a los centros de concentración, selección y contratación de aspirantes, cuyo punto más cercano — durante los primeros años del programa - lo constituyó la ciudad de México, sede que posteriormente se trasladó al estado de Guanajuato extendiéndose más la distancia respecto a Chiapas; 2) la difusión de que entre los requisitos que debían cumplir los aspirantes consistía en ser lectoescritores; y por último, 3) la interdependencia que la mano de obra chiapaneca tenía con las plantaciones de café, sin desestimar el papel del enganche y el sistema de deudas que el trabajador tenía con sus respectivos patrones. De ahí que geografía, perfil sociodemográfico de la población e interés económico de los grupos de poder local, acaecieron como factores estructurales en la retención de la mano de obra chiapaneca. ${ }^{1}$

Es en ese contexto cuando comenzó a generarse una diferenciación entre oaxaqueños y chiapanecos que hasta muy entrados los años 90 sus patrones de emigración se habían perfilado bastante distintos, en tanto los primeros habían logrado desde tiempo atrás un posicionamiento significativo en su inserción en el mercado de trabajo y vida cotidiana de Estados Unidos, los segundos mantenían una lógica de inserción laboral constreñida a los límites del estado, aun cuando en ambos grupos históricamente se habían caracterizado por ostentar atributos en común, consistentes en tener un fuerte componente indígena, además de estar insertos en un contexto de marginación y pobreza. Advertir tal situación llevó a 
Martínez (1999b) a realizar un análisis de los factores subyacentes que diferenciaban a dicho comportamiento migratorio, identificando los siguientes: 1) la participación o no en el programa bracero; y 2) la disponibilidad o no de terrenos agrícolas en sus respectivos ámbitos estatales. Del primer factor se han hecho ya los señalamientos, mientras que para el segundo se verificó que a diferencia del caso oaxaqueño, en que hacia los años 50 la tierra había sido totalmente repartida, localizándose regiones en donde la fragmentación de la misma había llegado a un grado tal que las sucesivas generaciones de herederos en lugar de recibir una parcela recibían un determinado número de surcos; en Chiapas el acceso a la tierra todavía constituía un asunto pendiente en la agenda de la movilización campesinoindígena, producto de la desigualdad en la tenencia de la misma, ${ }^{2}$ además de la disponibilidad de extensas superficies de terrenos federales, situaciones que jugaron un papel de retener a la población, siendo la tierra, hasta hoy día, objeto de colonización y su consecuente ampliación agropecuaria (Martínez 1999b).

Estos primeros factores constituyen unas de las causas fundamentales de la inserción tardía de Chiapas en la emigración internacional constituyéndose en la primera distinción que asume la entidad en el contexto nacional.

Después del programa bracero, 60 años más tarde los perfiles sociales de aquellos grandes grupos de población trabajadora de Chiapas habrían de registrar una serie de profundos cambios individuales, familiares y comunitarios; sólo para ilustrar tal situación, mientras en 1940 la tasa de analfabetismo llegaba a 75.0\% del total de la población, en 2005 había bajado a 21.3\%. Los indígenas bilingües hablantes de castellano en 1940 era $17.0 \%$, mientras que en 2005 ascendía a 61.2\% (Secretaría de Economía 1940; INEGI 2005).

\section{LA INCURSIÓN INTERNACIONAL}

Ciertas evidencias documentales indican que las primeras incursiones a Estados Unidos se dieron por parte de la población indígena en el primer quinquenio del decenio de los 90, específicamente por parte de la población de San Juan Chamula (Rus y Rus 1996, 2008), no 
sin antes ampliar sus territorios de incursión laboral en distintos lugares del sur-sureste y del centro del país. Asimismo, otros contingentes comenzaron a arribar en estados cercanos a la frontera norte constituidos por ejidatarios y aparceros localizados en las regiones de El Soconusco y Sierra a principios de la década de los años 90 debido a la caída en los precios internacionales del café y el aumento constante de trabajadores guatemaltecos en las plantaciones cafetaleras de Chiapas, que tampoco hallaban fuentes de trabajo en la cafeticultura de aquel país. Las poblaciones de trabajadores nacionales de las dos últimas regiones mencionadas comenzaron a emplearse como jornaleros agrícolas en los campos hortícolas de Nayarit y Sinaloa, para luego dar el salto a Estados Unidos. ${ }^{3}$

Con base en el censo de población levantado en 2000, en su Índice de intensidad migratoria por entidad federativa (CONAPO 2002) el Consejo Nacional de Población clasificó al estado de Chiapas en la categoría de muy baja emigración. Tiempo después, en 2005, el mismo organismo publicó Migración México-Estados Unidos. Panorama regional y estatal sin que la entidad chiapaneca sobresaliera en dicho análisis. Se infiere que la causa por la que se le clasificó a la entidad como de baja emigración reside en que la pregunta sobre un evento migratorio internacional de cualquier miembro de un hogar se hacía en referencia a si éste había ocurrido o no cinco años atrás, es decir, en 1995, por lo que siendo ese año de poca ocurrencia de eventos migratorios a Estados Unidos, la catalogación de la entidad efectivamente fue de muy baja intensidad migratoria; situación que realmente coincidía con el insignificante flujo de remesas a la entidad en ese año. Sin embargo, en la segunda publicación señalada, siendo su base de análisis la Encuesta Nacional de Empleo (ENE, INEGI 2004) levantada en 2002, específicamente del módulo de migración de la citada encuesta, CONAPO no tomó en cuenta lo que la misma fuente de información advierte (INEGI 2004) en el sentido de que «Si bien una de las limitantes de las encuestas es que su nivel de detalle geográfico no puede ser tan amplio como el de los censos o como el del conteo, las últimas encuestas demográficas proporcionan información sobre migración por entidad federativa, por lo menos para aquéllas en las que el fenómeno tiene mayor importancia» (INEGI 2004, resaltado del autor). De antemano indica que el tamaño 
muestral de la ENE fue distinto en función de la importancia que la emigración estaba cobrando en el plano estatal, y en el caso de Chiapas, en tanto el fenómeno no había tenido importancia en el levantamiento censal de 2000 por las razones señaladas, por tanto, tampoco después lo tuvo en el levantamiento de la Encuesta Nacional de Empleo (ENE). En síntesis, si bien — como se dijo - la primera clasificación se muestra coherente con otras evidencias estadísticas, como es el monto de remesas captadas por la entidad en 1995, la segunda publicación difiere con el creciente flujo de remesas que para el año 2002 ya habían comenzado a ser significativas, tal como se mostrará más adelante, así como lo que consignan otras fuentes de información como la Encuesta de Migración de la Frontera Norte (EMIF).

\section{LA EVIDENCIA CUANTITATIVA}

Los registros recientes sobre la emigración de chiapanecos indican que la presencia de éstos en Estados Unidos había aumentado de 2,434 personas en 1995 a 118,510 en 2006, en términos relativos esto representa un tránsito de $0.6 \%$ a $14.0 \%$ del total del flujo nacional en 2006 (EMIF 2006). Esta alza muestra de facto la contundente participación de esta entidad en la emigración de mexicanos a Estados Unidos. En dicha encuesta, los chiapanecos declaraban en $94.6 \%$ que el cruce de la frontera norte lo estaban haciendo por primera vez. Esto indica que año con año gran parte del flujo se había compuesto de población que iniciaba su cruce e inserción laboral en Estados Unidos. Con base en la cuantificación señalada se revela que en ese periodo se tuvo un crecimiento porcentual de 4,768.9\%, con una tasa de crecimiento media anual (TCMA) de 42.3\%; situación que convertía a Chiapas en una de las entidades más dinámicas en su internación a Estados Unidos que se haya registrado en México. Esos datos contrastan con lo ocurrido en el ámbito nacional en cuyo periodo se presentó un incremento porcentual de 96.4\%, con una TCMA de 6.3\% (EMIF 2006). En un reporte del Migration Policy Institute señala que aun cuando en Estados Unidos en 2008 se anunciaban ya severamente los síntomas de la recesión de 2009, el 14.2\% de la corriente mexicana hacia aquel país lo constituía los chiapanecos (MPI 2010). 
Lo anterior constituye un segundo rasgo distintivo que tiene la migración chiapaneca que consiste en perfilar una trayectoria migratoria de rápida expansión que supera los límites estatales con carácter temporal para dispersarse inmediatamente en los destinos nacionales anteriormente señalados y luego convertirse en una emigración internacional de considerable intensidad. Al cruzar indocumentadamente le impone estancias bastante prolongadas, y por tanto una menor circularidad que la que anteriormente ofrecían los destinos nacionales. Por ejemplo, EMIF reportó para 2007 que 82.0\% de los encuestados declaró su intención de quedarse en la Unión Americana el tiempo que pudiera.

El despunte que la emigración chiapaneca ha tenido se ha expresado también en el monto de aprehensiones de oriundos de este estado que la patrulla fronteriza de Estados Unidos ha realizado. Según la misma fuente (EMIF), las devoluciones pasaron de 4,731 en 1995 a 48,745 en 2005 , lo que significa un aumento porcentual de 930.0 con tasas anuales de 26.3\%; cifras en sentido contrario a las nacionales que para ese mismo periodo registraban un decremento de $-24.7 \%$. De acuerdo con lo expuesto, merece resaltar que mientras las tendencias nacionales en aprehensiones se encontraban totalmente a la baja, con excepción del año 2005, la de los chiapanecos iban a la alza con mayor agudeza en el año señalado. Esta situación nos permite emitir a manera de hipótesis que los contingentes chiapanecos se encuentran en mayor exposición de ser aprehendidos debido a lo reciente de sus incursiones en la Unión Americana, y por sus aún nacientes redes sociales de migración, que hasta el momento se han reducido a servir solamente como facilitadores o coyotes sin que se hayan generado formas superiores de apoyo mutuo en el lado estadounidense.

Según EMIF los puntos de cruces que usan los chiapanecos se distribuyen de la siguiente forma: Nogales, en Sonora (34.4\%), seguida por Agua Prieta, también de Sonora (13.8\%), y Tijuana, en Baja California (12.9\%), el resto de lugares son de no especificados (2006). Cabe destacar que Sásabe, localidad perteneciente al estado de Sonora, ocupa un prominente punto de cruce debido a mayores probabilidades de éxito para acceder a la 
Unión Americana a causa de ser un amplio territorio desértico, pero al mismo tiempo, por esa misma razón se convierte en el de mayor peligrosidad de fallecer por la deshidratación que esto conlleva, más aún cuando los migrantes son abandonados por el coyote o simplemente por perder el camino adecuado.

De acuerdo con registros de la Secretaría para el desarrollo de la frontera sur, del gobierno del estado de Chiapas, se habían contabilizado —en el primer semestre de 2010 - 15 casos de personas desaparecidas, y más 19 decesos cuyos cadáveres habían sido trasladados a sus lugares de origen de la entidad, lo que denota una fragilidad en el cruce y estancia (SPLFS 2010).

Producto de la ausencia de redes maduras, muchos chiapanecos han optado por el retorno voluntario, mediante el Programa de Repatriados instrumentado por la Dirección General de Protección y Asuntos Consulares dependiente de la Secretaría de Relaciones Exteriores (Cuadro 3).

Cuadro 3. Repatriaciones por principales entidades federativas, 2007-2008.

\begin{tabular}{|l|r|r|l|r|r|}
\hline \multicolumn{2}{|c|}{2007} & \multicolumn{3}{c|}{ 2008 } \\
\hline Entidades federativas & $\begin{array}{c}\text { Totales } \\
\text { Repatriados }\end{array}$ & \multicolumn{1}{c|}{$\begin{array}{l}\text { Totales } \\
\text { Repatriados }\end{array}$} & \multicolumn{1}{c|}{ \% } \\
\hline Puebla & 1,798 & 16.10 & Puebla & 2,355 & 16.49 \\
\hline Veracruz & 1,546 & 13.84 & Distrito Federal & 1,961 & 13.73 \\
\hline Chiapas & 1,366 & 12.23 & Estado de México & 1,545 & 10.82 \\
\hline D.F. & 1,284 & 11.49 & Sinaloa & 1,448 & 10.14 \\
\hline Oaxaca & 1,132 & 10.13 & Michoacán & 1,434 & 10.04 \\
\hline Guerrero & 943 & 8.44 & Guerrero & 1,298 & 9.09 \\
\hline Michoacán & 927 & 8.30 & Veracruz & 1,254 & 8.78 \\
\hline Estado de México & 784 & 7.02 & Oaxaca & 1,249 & 8.74 \\
\hline Hidalgo & 768 & 6.87 & Chiapas & 919 & 6.43 \\
\hline Sinaloa & 623 & 5.58 & Hidalgo & 822 & 5.75 \\
\hline Total & 11,171 & 100.00 & Total & 14,285 & 100.00 \\
\hline
\end{tabular}

Fuente: SRE, DGPAC 2008. Informe de actividades de la Dirección General de Protección y Asuntos Consulares. 
En 2007, 1,366 chiapanecos solicitaron su traslado de retorno, equivalente a $12.2 \%$ del total nacional, ubicándose en el tercer lugar de repatriados después de Puebla y Veracruz; reduciéndose su volumen un año más tarde a 919 (IME 2007). Cabe destacar que estas entidades coinciden en ser de las denominadas emergentes en la emigración internacional, lo que supone compartir en común esa ausencia de maduración de las redes sociales y del paisanaje en Estados Unidos.

\section{DISTRIBUCIÓN DE CHIAPANECOS EN ESTADOS UNIDOS}

Respecto a la distribución de los chiapanecos en Estados Unidos, en 2007, de acuerdo con el Instituto de los Mexicanos en el Exterior (IME), los consulados de Los Ángeles, Orlando, Atlanta, Washington y Miami, concentraban el mayor número de matrículas expedidas a chiapanecos con 10.7, 8.6, 7.5, 6.5 y 6.3\%, respectivamente. ${ }^{4}$ Información más reciente (SPLFS 2010) indica que los consulados establecidos en el estado de Arizona (Phoenix, Tucson, Douglas, Nogales, Yuma) han venido ganando mayor importancia, ya que para 2010 se habían registrado 1,504 chiapanecos, con mayor primacía en el de Phoenix.

Sin embargo, parece ser otra distinción más de los chiapanecos la de que de los consulados existentes en Estados Unidos los chiapanecos se registran en casi todos ellos, ya que de las 45 oficinas consulares de México en Estados Unidos, en 41 de ellas han solicitado su registro. Esto nos brinda una idea de la pronta expansión territorial de la migración chiapaneca a ese país, lo que hace de este fenómeno un pivote de búsqueda de nuevos mercados laborales en territorio norteamericano, al mismo tiempo que también revela una baja integración y cohesión territorial en ámbitos acotados de los Estados Unidos, propiciando así una dispersión geográfica (Cuadro 4). Esta situación denota que a pesar de que los emigrantes supuestamente tienen un lugar fijo donde llegar, en el que el estado de California sobresale en primer término, le sigue Florida y en tercero Arizona (EMIF 2007), no significa que sean lugares definitivos, por el contrario, parecen éstos plataforma de lanzamiento para otros destinos en la Unión Americana. 
Cuadro 4. Distribución territorial de chiapanecos en Estados Unidos: principales consulados, 2007.

\begin{tabular}{|l|r|}
\hline Estados & Porcentaje \\
\hline Los Ángeles & 10.6 \\
\hline Orlando & 8.5 \\
\hline Phoenix* & 7.8 \\
\hline Atlanta & 7.4 \\
\hline Washington & 6.4 \\
\hline Miami & 6.2 \\
\hline
\end{tabular}

*dato de 2009.

Fuente: Instituto de los Mexicanos en el Exterior, DGAC, SER, 2007.

Aun cuando líneas arriba se señale de lo incipiente en la estructuración de las redes chiapanecas, signos de cohesión identitaria han comenzado a ocurrir en la medida de que en distintas entidades de Estados Unidos los emigrantes han comenzado a agruparse, lo que puede develar una tendencia que se opone a la dispersión antes señalada que consiste en las formaciones de los primeros clubes de migrantes oriundos de Chiapas. En 2008, se tenían registrados cinco clubes de diferentes tamaños en número de miembros, unos con planteamientos de carácter estatal y otros de corte municipal. Sus diferentes propósitos van desde a) interés de mantener raíces y tradiciones; b) mejoramiento del bienestar de las poblaciones que se quedan en los lugares de origen y de los que se encuentran residiendo en los Estados Unidos; hasta de aspiraciones concretas como la de c) participar en el programa 3 x 1 de la Secretaría de Desarrollo Social del gobierno federal, así como d) enviar remesas para propiciar mejores condiciones de trabajo y desarrollo del campo chiapaneco. Dos de los cinco clubes se encuentran en California (Los Ángeles, San Diego), uno en el estado de Iowa, otro en el de Utah y el más reciente en Texas (IME 2010). Mención especial merece el caso de Utha, en donde se fundó desde 1996 el «Club de Chiapanecos Unidos en Utha», en 
Salt Lake City, por una chiapaneca oriunda del municipio de Suchiate, llamada Rhosbita Barker. Más tarde, el 9 de junio de 2010, teniendo como sede la Casa Chiapas en West Valley, Utha, se conformó la Federación de clubes de chiapanecos en EUA, con nueve grupos ubicados en los estados de Florida y California. Los de California se denominan San Martín de Jitotol y San Miguel, ubicados en el condado de los Ángeles, básicamente integrados por oriundos del municipio de Tonalá; el Club Jaguares, el Club Lacandones, por los de los municipios de Tapachula y Tuxtla Gutiérrez; el Club los Parachicos de Chiapa de Corzo, el Club Chiapanecos Unidos, el Club Los Altos y Familia Ruiz ubicados en el estado de Utha, y el Club Acapetahua, de oriundos del mismo municipio radicados en el estado de la Florida (SPLFS 2010). Sin duda, detrás de estos esfuerzos de unión priva el espíritu de cooperación y de vinculación con sus municipios y gobierno de origen, así como emprender proyectos de desarrollo agropecuario. Es probable que posteriormente puedan unirse otros clubes radicados en Texas, Georgia, Carolina del Norte, Iowa y recientemente otro conformado en Tennessee (SPLFS 2010).

\section{CARACTERÍSTICAS SOCIODEMOGRÁFICAS}

En relación con la participación de hombres y mujeres en el flujo chiapaneco, si bien la corriente es mayoritariamente masculina, resulta relevante que $22.5 \%$ se componga por mujeres chiapanecas, ligeramente superior a la media nacional, que es de $22.1 \%$ (CONAPO, ENADID 2006; Cuadro 5). En complemento de lo anterior, cabe destacar que dicha estimación resulta ser conservadora, ya que si se toman los registros del IME, la preponderancia de mujeres resulta ser mayor, ya que la presencia de ellas en dicho flujo a EUA asciende a $31.7 \%$ (IME 2007). Esta situación sin duda alguna pone a la migración chiapaneca en un nivel de avanzada en términos de la incorporación femenina, ya que de acuerdo con los patrones de emigración mexicana a Estados Unidos, se ha constatado la existencia de un diferencial de tiempo considerable entre las primeras incursiones masculinas y sus subsecuentes femeninas, por lo que en el caso chiapaneco esta situación ocurre en un breve lapso de tiempo, igualando o superando así, en pocos años, a sus contrapartes femeninas nacionales. Otra característica más de este flujo chiapaneco. 
Cuadro 5. Chiapas-México en Estados Unidos, según sexo, 2006.

\begin{tabular}{|l|r|r|}
\hline Sexo & Porcentaje nacional & Porcentaje Chiapas \\
\hline Hombre & 77.93 & 77.5 \\
\hline Mujer & 22.16 & 22.5 \\
\hline Total & 100.00 & 100.00 \\
\hline
\end{tabular}

Fuente: CONAPO, ENADID 2006.

Respecto al parentesco, cobra relevancia destacar que en el caso que atañe son los hijos/as los que están asumiendo la responsabilidad de la emigración, ya que ellos representan la mitad del flujo, seguido del esposo correspondiéndole un tercio de la corriente (Cuadro 6).

Cuadro 6. Chiapanecos en Estados Unidos de Norteamérica según parentesco, 2006.

\begin{tabular}{|l|r|r|}
\hline Parentesco & \multicolumn{1}{|c|}{$\begin{array}{c}\text { Porcentaje } \\
\text { nacional }\end{array}$} & $\begin{array}{c}\text { Porcentaje } \\
\text { Chiapas }\end{array}$ \\
\hline Jefe o jefa & 12.07 & 12.5 \\
\hline Esposo(a) o compañero & 6.73 & 32.5 \\
\hline Hijo(a) & 20.03 & 50.0 \\
\hline Padre o madre & 0.59 & 0.0 \\
\hline Abuelo(a) & 0.02 & 0.0 \\
\hline Hermano(a) & 0.78 & 0.0 \\
\hline Nieto(a) & 1.08 & 0.0 \\
\hline Otro parentesco & 3.04 & 5.0 \\
\hline No tiene parentesco & 0.66 & 0.0 \\
\hline No especificados & 55.01 & 0.0 \\
\hline Total & 100.00 & 100.00 \\
\hline
\end{tabular}

Fuente: CONAPO, ENADID 2006.

Esta situación contrasta con lo observado a nivel nacional, ya que a esa escala la participación de los hijos/as se reduce a $20.0 \%$, y la del esposo a $6.7 \%$. Esta situación sugiere pensar que en el caso chiapaneco son los hijos los que ante un escenario socioeconómico incierto que exhibe la entidad conllevan a presentar una falta de 
expectativas de mejorar sustancialmente sus niveles de vida, y acuden a la migración internacional como mecanismo de obtención de recursos en montos más redituables y en tiempos menos prolongados que en Chiapas, aparte de lo que para ellos significa la experiencia varonil de la llamada aventura migratoria.

El perfil educativo captado a través de los registros del IME anuncian una migración cuya población al menos tiene la primaria con certificado, que representa $23.2 \%$ del total de registrados; mientras que lo más sobresaliente es la población que cuenta con secundaria completa, que asciende a la mitad del total de registros (49\%), y una apreciable proporción de $18 \%$ que tiene preparatoria terminada, sin dejar de mencionar que del conjunto $2.6 \%$ cursó el nivel profesional, y 3.0\% no cuenta con estudios de ningún nivel (Figura 1).

Figura 1. Escolaridad de los chiapanecos en los Estados Unidos, año 2007.

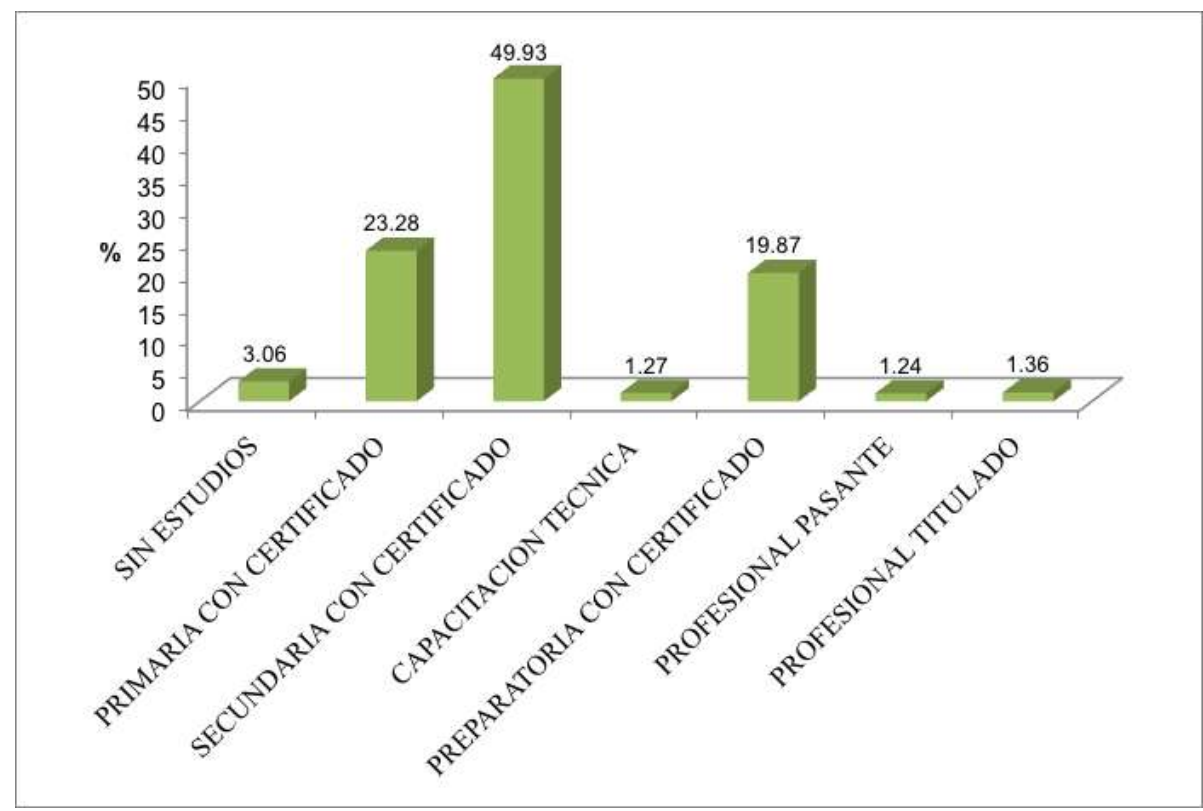

Fuente: Instituto de los Mexicanos en el Exterior, DGAC, SRE 2007.

Esto nos indica que la migración chiapaneca está constituida por una población 
considerable que inició sus estudios primarios y alcanzó concluir su secundaria, además de otro porcentaje apreciable que consiguió el nivel preparatoria; por lo que refiere entonces a una población que emigra aun contando con una trayectoria individual educativa, que ante la baja expectativa de contar con empleos lo suficientemente remunerados deciden truncar sus estudios. Esta situación debe contextualizarse en el escenario de un país como México en el que prevalece un déficit en la oferta de educación media superior y superior por parte del sector gubernamental, y la consecuente participación de la iniciativa privada que desde hace varias décadas se ha difundido en el territorio nacional cubriendo esos vacíos educativos bajo una lógica empresarial.

Esta situación en general coincide con algunos datos provenientes de EMIF (2007), en relación con que dicha fuente señala que $30.0 \%$ ha concluido la primaria, aunque debe señalarse que con los otros niveles de educación ciertamente existen diferencias.

Respecto del origen de los emigrantes chiapanecos también es diverso. Según los registros de expedición de credenciales giradas por IME, pueden apreciarse oriundos de casi todos los municipios de la entidad que han acudido a esa instancia para la obtención de una matrícula de alta seguridad. En el desglose por regiones podemos apreciar, según IME, la preponderancia que tiene la región Soconusco cuya presencia absorbe poco más del tercio del total de registrados en ese país; en segundo lugar, un grupo de regiones en igualdad de participación: Fronteriza, Centro, Istmo-Costa y Sierra, con porcentajes de 13.2, 10.8, 10.3 y 8.5 , respectivamente (Figura 2). Enseguida se encuentra otro grupo de regiones de menor intensidad, como las de Frailesca, Selva, Norte y Altos. 
Figura 2. Porcentaje de chiapanecos por regiones en EUA, año 2007.

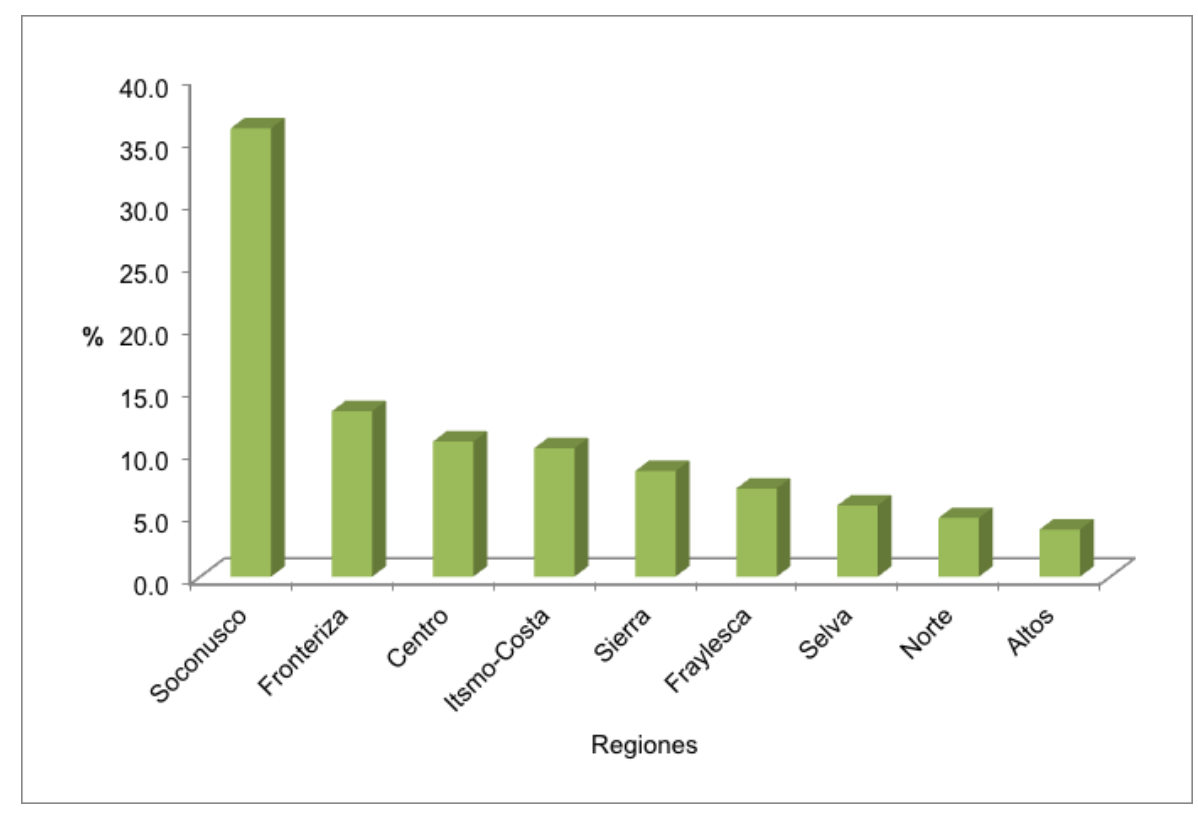

Fuente: Instituto de los Mexicanos en el Exterior, DGAC, SRE 2007.

En el ámbito municipal, la preponderancia la cobran determinados espacios municipales, que para fines del establecimiento de políticas públicas interesan reconocer (Cuadro 7). Por ejemplo, en la región Soconusco el peso mayor reside en el municipio de Tapachula, que absorbe un tercio de la emisión de migrantes; mientras que para la región Sierra es el municipio de Motozintla, participando con más de un tercio (36.9\%), seguido de Siltepec con $16.9 \%$, y Amatenango de la Frontera con 16.2\%; por su parte, en la región Fronteriza Comalapa se hace notable con 28.4\%; La Trinitaria con 23.2\%; Las Margaritas con 14.5\%; Comitán con $12.9 \%$ y Chicomuselo con $10.3 \%$. De la región Centro, Tuxtla Gutiérrez destaca con casi un tercio de la población emigrante (30.0\%); enseguida se encuentran tres municipios más cuyo porcentaje los hace destacar: Tecpatán, Jiquipilas y Cintalapa, con $13.4,10.4$ y $10.0 \%$, respectivamente. 
Cuadro 7. Municipios de mayor importancia en la migración a EUA, según región, 2007.

\begin{tabular}{|c|c|c|}
\hline Región & Municipios & Porcentaje \\
\hline Soconusco & Tapachula & 33.3 \\
\hline \multirow{3}{*}{ Sierra } & Motozintla & 36.9 \\
\hline & Siltepec & 16.9 \\
\hline & Amatenango de la frontera & 16.2 \\
\hline \multirow{5}{*}{ Fronteriza } & Frontera Comalapa & 28.4 \\
\hline & La Trinitaria & 23.2 \\
\hline & Las Margaritas & 14.5 \\
\hline & Comitán de Domínguez & 12.9 \\
\hline & Chicomuselo & 10.3 \\
\hline \multirow{4}{*}{ Centro } & Tuxtla Gutiérrez & 30.0 \\
\hline & Tecpatán & 13.4 \\
\hline & Jiquipilas & 10.4 \\
\hline & Cintalapa & 10.0 \\
\hline \multirow{3}{*}{ Istmo-Costa } & Tonalá & 42.0 \\
\hline & Pijijiapan & 37.0 \\
\hline & Arriaga & 21.0 \\
\hline \multirow{5}{*}{ Frailesca } & Villacorzo & 39.6 \\
\hline & Villaflores & 24.8 \\
\hline & La Concordia & 21.7 \\
\hline & Ángel Albino Corzo & 13.3 \\
\hline & Montecristo de Guerrero & 0.5 \\
\hline \multirow{3}{*}{ Selva } & Palenque & 42.6 \\
\hline & Ocosingo & 25.3 \\
\hline & Salto de Agua & 14.2 \\
\hline \multirow{4}{*}{ Norte } & Reforma & 18.9 \\
\hline & Juárez & 11.8 \\
\hline & Pichucalco & 11.6 \\
\hline & Ostuacán & 10.9 \\
\hline \multirow{2}{*}{ Altos } & Chamula & 25.6 \\
\hline & San Cristóbal de Las Casas & 21.5 \\
\hline
\end{tabular}

Fuente: Instituto de los Mexicanos en el Exterior, DGAC, SRE 2007.

Mientras que los tres municipios que integran la región Istmo-Costa, todos participan con su respectiva importancia en el flujo laboral: Tonalá con 42.0\%, Pijijiapan con 37.0\%, y Arriaga con 21.0\%. En la región Frailesca, 4 de sus 5 municipios tienen relevancia en la migración: en primer lugar Villacorzo, con 36\%, en segundo Villaflores con 24.8; La Concordia, con 21.7, y Ángel Albino Corzo con 13.3\%; solamente Montecristo de Guerrero resulta tener una participación casi nula al representar $0.5 \%$ del total de migrantes de la 
región. De la extensa región Selva, es muy notable la participación de Palenque con 42.6\%, a cierta distancia le sigue Ocosingo con una cuarta parte del total regional (25.3\%), y por último Salto de Agua con 14.2\%. De los numerosos municipios de la región Norte (24), destacan 4 de ellos, que son Reforma con 18.9\%, Juárez con 11.8, Pichucalco con 11.6, y Ostuacán con 10.9\%. Por último, de la zona mayoritariamente indígena del estado, que es la región Altos, dos municipios sobresalen con relevancia: Chamula, con una cuarta parte de la región (25.6\%), y San Cristóbal con 21.5\%.

Así, de los 118 municipios existentes en la entidad en el momento del registro de la información, en 115 está presente la emigración, quedando solamente exentos de esta corriente los municipios de Aldama, San Andrés Duraznal y Santiago El Pinar. Al contrastar esta información proveniente del IME con la proveniente de la EMIF, se tiene que esta última fuente señala a 95 los municipios participantes. Se puede plantear a manera de hipótesis que el IME puede estar captando un mayor rango de municipios en tanto exige evidencias sobre el origen del solicitante de una matrícula consular.

Para fines de confiabilidad de la fuente de información proveniente del IME, la notable participación del municipio de Chamula en los registros de expedición de matrículas consulares resulta ser una valiosa evidencia de que los chiapanecos en general han acudido a esta instancia gubernamental independientemente de su adscripción étnica, nivel educativo y sus respectivas habilidades lingüísticas que de ella se derivan, ya que podría pensarse que quienes acuden a solicitar una matrícula consular podrían ser únicamente los pertenecientes a la población mestiza, o en su defecto personas con algún nivel de estudios cursado. De ahí que la base de datos provista por esta fuente contenga una considerable representatividad - al menos - para el flujo de migrantes chiapanecos.

Con base en los municipios de origen, nos remite a señalar que esta migración se encuentra ampliamente difundida en distintos espacios sociales de la entidad, en el que intervienen regiones y municipios de mayor desarrollo como El Soconusco y su municipio de 
Tapachula, o bien la región Centro y su municipio de Tuxtla Gutiérrez, como también otras regiones de mucho menor desarrollo como la región Altos y uno de sus municipios indígenas como el de Chamula, y otro de perfil multicultural como es el de San Cristóbal de Las Casas. De ahí que la caracterización sociodemográfica revelada en la emigración internacional atraviese a las distintas regiones, municipios y perfiles étnicos.

\section{LAS REMESAS}

La captación de remesas familiares en Chiapas había pasado de ocupar el lugar 27 en 1995 a la posición 15 en 2001 para alcanzar en 2005 el lugar 11, posición ocupada hasta la fecha. De acuerdo con el siguiente Cuadro 8, podemos apreciar una fase de iniciación poco significativa que va de 1995 a 2000, en donde se estima un promedio de 13 millones de dólares de poco impacto a nivel del producto interno bruto de la entidad (PIB), a una fase de crecimiento altamente significativo de 2001 a 2006, con una cúspide en el último año del periodo en el que se llegó a captar 943.6 millones de dólares; y enseguida una fase de desaceleración que inicia de 2007 a 2009 cuando desciende en el último año a un valor de 472.2 millones de dólares, cifra similar a la que se tuvo en 2003.

El flujo de remesas ha llegado a representar en 2006 el $6.7 \%$ del PIB de la entidad, valor que representa a más del doble del que corresponde a nivel país. En la cúspide de su monto también llegó a representar 94.0\% por concepto de salarios del sector formal en Chiapas y han sido superiores al total del valor de la producción de los principales cultivos del sector agropecuario del estado (López 2009). En Chiapas, como en México, a partir del año 2006 comienza a declinar la importancia del ingreso de divisas vía remesas debido fundamentalmente a la desaceleración de la economía estadounidense que redujo la contratación laboral en términos de horas-hombre en los mercados de trabajo de aquel país. 
Cuadro 8. Chiapas, Monto de remesas familiares anuales, 1990-2009 (millones de dólares).

\begin{tabular}{|c|c|c|c|c|c|}
\hline \multirow{2}{*}{ Años } & Nacional & \multirow{2}{*}{ TCMA } & Chiapas & \multirow{2}{*}{ TCMA } & \multirow{2}{*}{$\%$} \\
\hline & Remesas familiares & & Remesas familiares & & \\
\hline 1990 & 2,494 & --- & --- & ---- & --- \\
\hline 1991 & 2,660 & 6.7 & 0 & 0.0 & 0.0 \\
\hline 1992 & 3,070 & 15.4 & 0 & 0.0 & 0.0 \\
\hline 1993 & 3,333 & 8.6 & 0 & 0.0 & 0.0 \\
\hline 1994 & 3,475 & 4.2 & 0 & 0.0 & 0.0 \\
\hline 1995 & 3,673 & 5.7 & 20 & 0.0 & 0.5 \\
\hline 1996 & 4,224 & 15.0 & n.d. & 0.0 & 0.0 \\
\hline 1997 & 4,865 & 15.2 & n.d. & 0.0 & 0.0 \\
\hline 1998 & 5,627 & 15.7 & n.d. & 0.0 & 0.0 \\
\hline 1999 & 5,910 & 5.0 & n.d. & 0.0 & 0.0 \\
\hline 2000 & 6,573 & 11.2 & 13 & 0.0 & 0.2 \\
\hline 2001 & 8,895 & 35.3 & 56 & 0.0 & 0.6 \\
\hline 2002 & 9,815 & 10.3 & n.d. & 0.0 & 0.0 \\
\hline 2003 & 13,650 & 39.1 & 439 & 0.0 & 3.2 \\
\hline 2004 & 16,730 & 22.6 & 596 & 35.6 & 3.6 \\
\hline 2005 & 20,284 & 21.2 & 772 & 29.6 & 3.8 \\
\hline 2006 & 23,742 & 17.1 & 944 & 22.2 & 4.0 \\
\hline 2007 & 23,970 & 1.0 & 906 & -4.0 & 3.8 \\
\hline 2008 & 25,145 & 4.9 & 800 & -11.7 & 3.2 \\
\hline 2009 & 21,181 & -15.76 & 606 & -24.3 & 2.9 \\
\hline
\end{tabular}

n.d.= no disponible.

Fuente: Banco de México, Sistema de Información Económica (SIE), Indicadores Económicos, varios años; y página WEB: www.banxico.org.mx.

Con base en datos recientes del último Censo Agropecuario levantado en 2007, podemos advertir la importancia que tiene la captación de remesas para las regiones de Chiapas. De acuerdo con la Figura 3, la distribución del flujo monetario se presenta principalmente en tres regiones tal como sigue: en primer lugar se encuentra la región Fronteriza, en segundo lugar está la región de la Sierra, en tercer lugar El Soconusco. Se aprecian dos regiones más 
que están a considerable distancia de las primeras; estas son: la región Selva y la región Altos. Por último se encuentran las regiones Frailesca, Norte e Istmo-Costa. Como se ha visto y veremos adelante, esta situación guarda una relación directa con la intensidad migratoria de las regiones y municipios de la entidad.

Figura 3. Unidades de producción cuyos productores reciben dinero desde otro país según envíos de familiares, 2007.

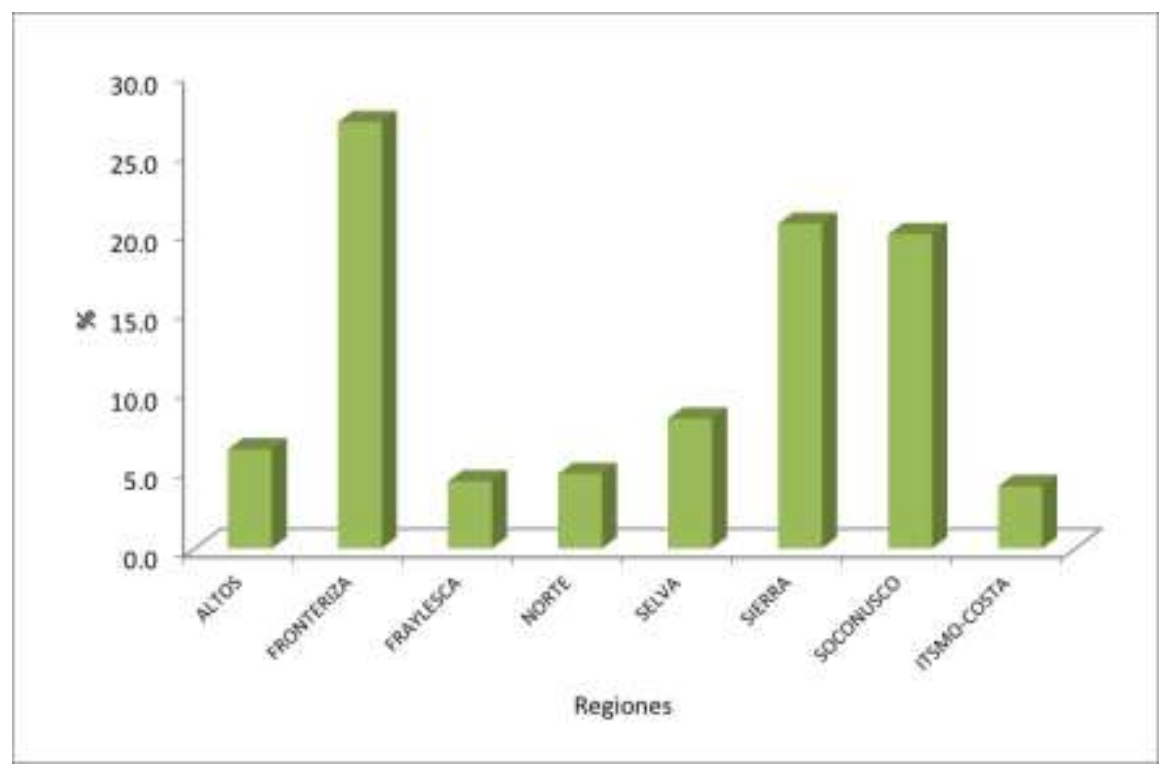

Fuente: INEGI. Estados Unidos Mexicanos. Censo Agropecuario 2007, VIII Censo Agrícola, Ganadero y Forestal. Aguascalientes, Ags. 2009.

Sin embargo, al interior de las regiones la distribución a nivel municipal guarda distintos comportamientos ya que en varios de los casos son determinados municipios los que sobresalen; en otros, como en el caso de las regiones con menores municipios integrantes (Istmo-Costa; Frailesca) la distribución es más homogénea.

Por otra parte, ante la ausencia de estudios pormenorizados, no se ha podido cuantificar los impactos económicos de las remesas en Chiapas, más allá de sus expresiones visuales 
mediante la internación de vehículos provenientes de EUA en el medio rural y la edificación de casas-habitación de estilos que van desde el mejoramiento modesto de la vivienda hasta la ostentosidad apabullante, presumiblemente derivada no solamente de la recepción de remesas, sino de los dividendos que deja el coyotaje local cuyo auge le ha permitido constituirse en un modo de vida profesional a nivel de las microrregiones de Chiapas.

Usos marginales de las remesas se han presentado a través de la participación en el programa 3 x 1 de SEDESOL. En 2006 se lograron reunir un millón de pesos para canalizarlos en 14 proyectos productivos, que con las aportaciones de los gobiernos estatal y federal se integraron 4 millones, ejecutados en los municipios de Ocozocuatla, Tuxtla Gutiérrez, Villacorzo, Villaflores, Siltepec, Teopisca, Tecpatán y Tuzantán. Todos ellos municipios mestizos y, por tanto, no necesariamente los más marginados. ${ }^{5}$ En lo que se refiere a los últimos años, en los ejercicios de 2008 a 2010, el número de acciones ha sido modesto, ya que en el primer año se llevaron a cabo 4 proyectos; en 2009 fueron 8; y en 2010 se redujo de nuevo a 4 (Cuadro 9).

Cuadro 9. Chiapas: programa 3 x 1 para migrantes: 2008-2010.

\begin{tabular}{|c|c|c|c|c|c|c|c|c|c|c|c|}
\hline \multirow{3}{*}{$\begin{array}{l}\text { Progra- } \\
\text { mas }\end{array}$} & \multicolumn{11}{|c|}{ Ejercido 2008-2010 } \\
\hline & \multirow{2}{*}{$\begin{array}{l}\text { Ejer- } \\
\text { cicio }\end{array}$} & \multirow{2}{*}{$\begin{array}{l}\text { Accio- } \\
\text { nes }\end{array}$} & \multicolumn{2}{|c|}{ No. benef. } & \multirow[b]{2}{*}{ Total } & \multirow[b]{2}{*}{$\begin{array}{c}\text { Fede- } \\
\text { ral }\end{array}$} & \multirow[b]{2}{*}{$\begin{array}{c}\text { Esta- } \\
\text { tal }\end{array}$} & \multirow[b]{2}{*}{$\begin{array}{l}\text { Sub- } \\
\text { total }\end{array}$} & \multirow[b]{2}{*}{$\begin{array}{l}\text { Aport. } \\
\text { Benef. }\end{array}$} & \multirow[b]{2}{*}{ Mpal. } & \multirow[b]{2}{*}{ Total } \\
\hline & & & $\mathbf{H}$ & $\mathbf{M}$ & & & & & & & \\
\hline \multirow{3}{*}{$\begin{array}{l}3 \times 1 \\
\text { para } \\
\text { migran- } \\
\text { tes }\end{array}$} & 2008 & 4 & 129 & 59 & 188 & 3.0 & 3.0 & 6.0 & 2.0 & 0.0 & 8.0 \\
\hline & 2009 & 8 & 1,117 & 614 & 1,731 & 3.9 & 3.9 & 7.8 & 2.8 & 0.0 & 10.7 \\
\hline & 2010 & 4 & 494 & 477 & 971 & 3.0 & 3.0 & 6.0 & 2.2 & 0.69 & 8.9 \\
\hline \multicolumn{2}{|r|}{ Totales } & 16 & 1,740 & 1,150 & 2,890 & 9.9 & 9.9 & 19.8 & 7.0 & 0.69 & 27.6 \\
\hline
\end{tabular}

Fuente: Secretaría de Desarrollo y Participación Social (SDyPS). Programas sociales convenidos, Ramo Administrativo 20. Gobierno del estado de Chiapas.

En el cuadro se aprecia que entre los tres años se han aplicado 27.6 millones básicamente 
provenientes de los presupuestos federal y estatal, situación que evidencia un ligero aumento anual en relación con 2006. Cabe destacar que es a partir de 2010 cuando, también modestamente, comienzan a participar con financiamiento algunos ayuntamientos municipales. Los contenidos de los proyectos han sido de diverso tipo, van desde los que se orientan al ámbito productivo en ganadería y agricultura o agroindustrial, hasta los de carácter social como la pavimentación de calles, construcción de albergues o centros de capacitación. Cabe señalar que lo reducido del número de proyectos, al menos para el caso del año 2010, más que a una falta de iniciativa por parte de las comunidades de migrantes ha sido básicamente por la escasez de fondos presupuestales de la parte gubernamental, ya que se recibieron 30 propuestas, de las cuales, solamente 4 fueron seleccionadas. Hasta ahora, los municipios recientemente beneficiados con el programa 3 x 1 han sido Pijijiapan, Ocozocuatla, Tuzantán, Venustiano Carranza, Teopisca, Chiapa de Corzo, Villaflores, Berriozabal, Suchiate, Teopisca, Ocosingo, Chilón, San Juan Cancuc, y Santiago El Pinar (Secretaria de Desarrollo y Participación Social 2010).

\section{CARACTERÍSTICAS SOCIALES DE LOS LUGARES DE ORIGEN}

En lo que se refiere a las condiciones sociales de los municipios de origen, podemos considerar que se esboza un patrón de asociación entre determinadas condiciones de vida de las regiones y municipios y la tendencia a emigrar. En primer lugar, abordaremos las expresiones regionales. Con base en el siguiente Cuadro 10 podemos apreciar, por ejemplo, la región de El Soconusco, siendo la de mayor intensidad migratoria que la ubica en primer lugar, ostenta un segundo lugar en el Índice de Desarrollo Humano (IDH), así como uno de los últimos lugares $\left(8^{\circ}\right)$ en el índice de marginación (IM), situación que indica que en el contexto estatal, esta región se ubica en condiciones mejorables.

La región Istmo-Costa, que ostenta las mejores condiciones del estado con un alto desarrollo humano y el más bajo im ( $9^{\circ}$ lugar), tiene una intensidad migratoria que se ubica en el cuarto lugar. La región Centro se ubica en el tercer lugar en el IDH, su IM no es tan alto $\left(7^{\circ}\right.$ lugar), mientras su posición migratoria se ubica en tercer lugar. Otro ejemplo 
contrastante reside en la región Altos, cuyo IDH es el más bajo, y la que tiene el más alto IM, encontrándose en el noveno lugar en su posición migratoria. Otra región especial es la Sierra, cuyo IDH la ubica en el quinto lugar, su IM la sitúa en el sexto lugar con una posición en la migración que la sitúa en el quinto lugar en el contexto estatal. Las regiones Selva y Norte, con bajos niveles de desarrollo humano y altos niveles de marginación, tienden a igualarse con el comportamiento de la región Altos; mientras que la región Frailesca tiene más similitud a la región Sierra.

Cuadro 10. Índice de Desarrollo Humano por regiones 2000 y 2005 y posición migratoria 2006.

\begin{tabular}{|l|c|c|c|c|c|}
\hline Regiones 2005 & IDH 2005 & $\begin{array}{c}\text { Posición } \\
\text { IDH-2005 }\end{array}$ & IM 2005 & $\begin{array}{c}\text { Posición } \\
\text { en IM }\end{array}$ & $\begin{array}{c}\text { Posición } \\
\text { migratoria } \\
\text { 2000 }\end{array}$ \\
\hline Istmo-Costa & 0,7760 & 1 & 0,0427 & 9 & 4 \\
\hline Soconusco & 0,7631 & 2 & 0,3789 & 8 & 1 \\
\hline Centro & 0,7308 & 3 & 0,4870 & 7 & 3 \\
\hline Frailesca & 0,7287 & 4 & 0,9346 & 4 & 6 \\
\hline Sierra & 0,7174 & 5 & 0,6940 & 6 & 5 \\
\hline Fronteriza & 0,7060 & 6 & 0,7839 & 5 & 2 \\
\hline Norte & 0,7020 & 7 & 0,9428 & 3 & 8 \\
\hline Selva & 0,6710 & 8 & 1,4727 & 2 & 7 \\
\hline Altos & 0,6250 & 9 & 1,6315 & 1 & 9 \\
\hline
\end{tabular}

Fuente: PNUD 2005. Índice de Desarrollo Humano (IDH); CONAPO 2005, Índice de Marginación (IM).

La región Fronteriza sobresale en relación con que sus indicadores no siguen un patrón como el que se ha venido perfilando en las regiones descritas, es decir, al tiempo que asume el segundo lugar en posición migratoria, su IDH la ubica en el $6^{\circ}$ lugar y su IM la sitúa en el $5^{\circ}$ lugar, esto es, siendo de alta intensidad migratoria, exhibe un regular desarrollo humano con una tendencia a un alto índice de marginación. En síntesis, si se omitiera la situación que guarda la región Fronteriza sería de interés explorar lo que parece presentarse aquí a nivel regional en el sentido de que se perfila un patrón de comportamiento migratorio 
regional, que consiste en que los espacios regionales que ostentan menores adversidades sociales parecen facilitar una mayor incorporación de su población en el flujo de la emigración internacional. Estas hipótesis concuerdan con los hallazgos señalados en el Informe de Desarrollo Humano 2007, en el sentido de «... que los migrantes en México no provienen de los hogares con mayor pobreza, $[\ldots]$ de manera que los hogares más pobres y los más ricos tienen menores probabilidades de tener un migrante» (PNUD 2007: 3).

En el análisis a nivel municipal, si se toman a aquellos municipios cuya participación en los registros del IME es igual o mayor a 10 puntos porcentuales, encontramos a municipios de muy alta y alta marginación, junto con pocos de media marginación, así como también con municipios de baja y muy baja marginación, es decir, a primera vista pareciera que participaran por igual casi todos los municipios con independencia de su índice de marginación. Es cierto que la mayoría de los municipios en las dos primeras categorías mencionadas son mayoría, sin embargo, a nivel del volumen con el que participan —como se ha dicho- están los municipios de Tapachula y Tuxtla Gutiérrez, mismos que sobresalen en el ámbito no solamente regional sino estatal, por lo que el efecto del número de municipios de alta y muy alta marginación de los primeros queda compensado por el efecto de la intensidad de los segundos.

Con el propósito de favorecer una mejor comprensión de las intersecciones entre el proceso de migración, índice de desarrollo humano e índice de marginación, se procedió a generar un índice de migración municipal que consiste en ponderar el número estimado de emigrantes en relación con sus tamaños de población respectiva. De esta forma, se establecieron las siguientes categorías: municipios de muy alta migración, alta migración, baja migración, muy baja migración.

Con base en ello, presentamos visualmente la relación que guarda el índice de intensidad migratoria municipal con sus correspondientes índices de desarrollo humano y de marginación, con resultados que aun siendo de carácter exploratorio marcan una clara 
tendencia que asocia índice de migración y condiciones sociales.

\section{MUNICIPIOS DE MUY ALTA MIGRACIÓN}

De acuerdo con las gráficas siguientes $(4,5,6$ y 7), y tomando como parámetros de referencia comparativa los correspondientes al estado de Chiapas, apreciamos en la primera Figura 4 la serie de municipios de muy alta migración, en donde sobresalen en los primeros cinco lugares municipios mayoritariamente de El Soconusco, alternando con otro de la región Sierra (Mazapa).

Figura 4. Chiapas: índice de desarrollo humano e índice de marginación, según intensidad migratoria muy alta 2007.

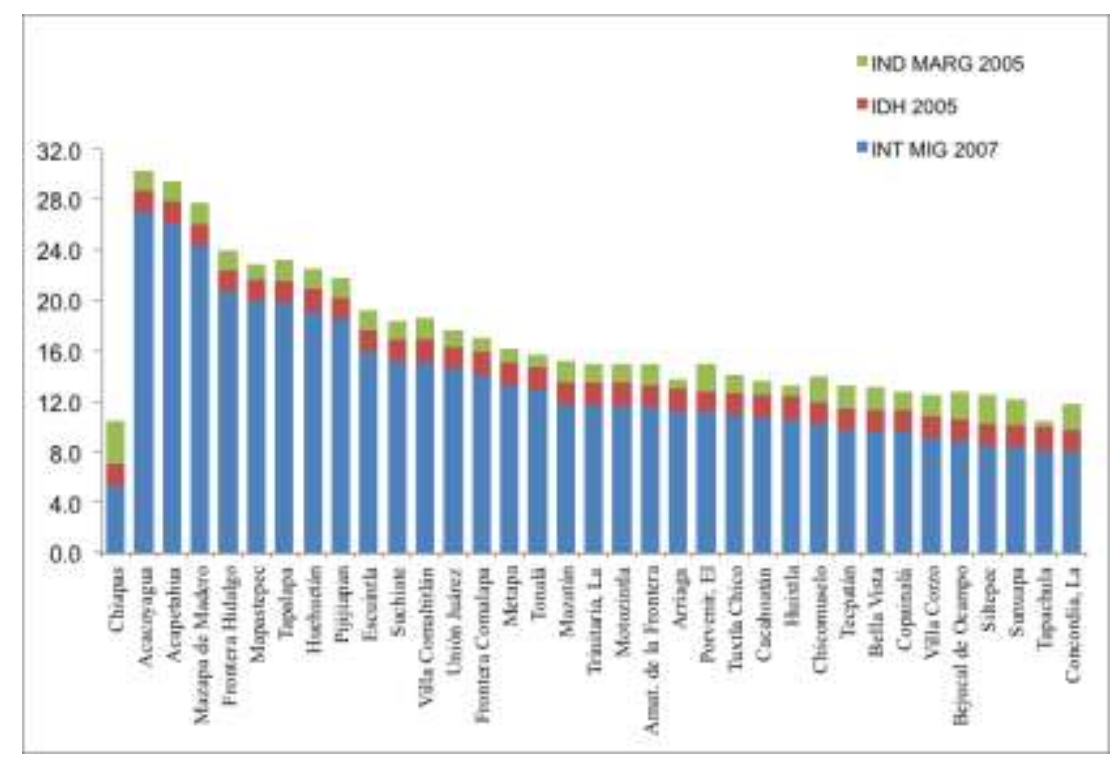

Fuente: CONAPO 2005-2007, PNUD 2007, IME 2006-2007.

En el resto de municipios también sobresale la región antes mencionada, lo que indica que siendo El Soconusco una región de bienestar se asocia con su índice de migración que la ubica en la categoría de muy alta intensidad migratoria. Como puede apreciarse en la misma Figura, la distinción entre los municipios de la serie solamente reside en el tamaño 
de su correspondiente índice de migración, ya que el del índice de desarrollo humano y el de marginación sus tamaños correspondientes son relativamente similares, con ligera excepción de cinco municipios: El Porvenir, Chicomuselo, Bejucal de Ocampo, Siltepec y Sunuapa, los cuatro primeros de la región Sierra y el último de la región Norte. Es decir, de 34 municipios catalogados de muy alta migración, solamente cinco - los cuales tienen menor intensidad dentro de dicha categoría - se apartan por su mayor índice de marginación; en el resto se aprecian valores similares incluyendo el índice de desarrollo humano, por lo que se infiere que en este caso la mayoría de municipios al tiempo que tienen una intensidad migratoria muy alta son los que presentan mejores niveles de bienestar.

\section{MUNICIPIOS DE ALTA MIGRACIÓN}

De acuerdo con la siguiente Figura 5, se observa que la categoría de intensidad migratoria

Figura 5. Chiapas: índice de desarrollo humano e índice de marginación, según intensidad migratoria alta 2007.

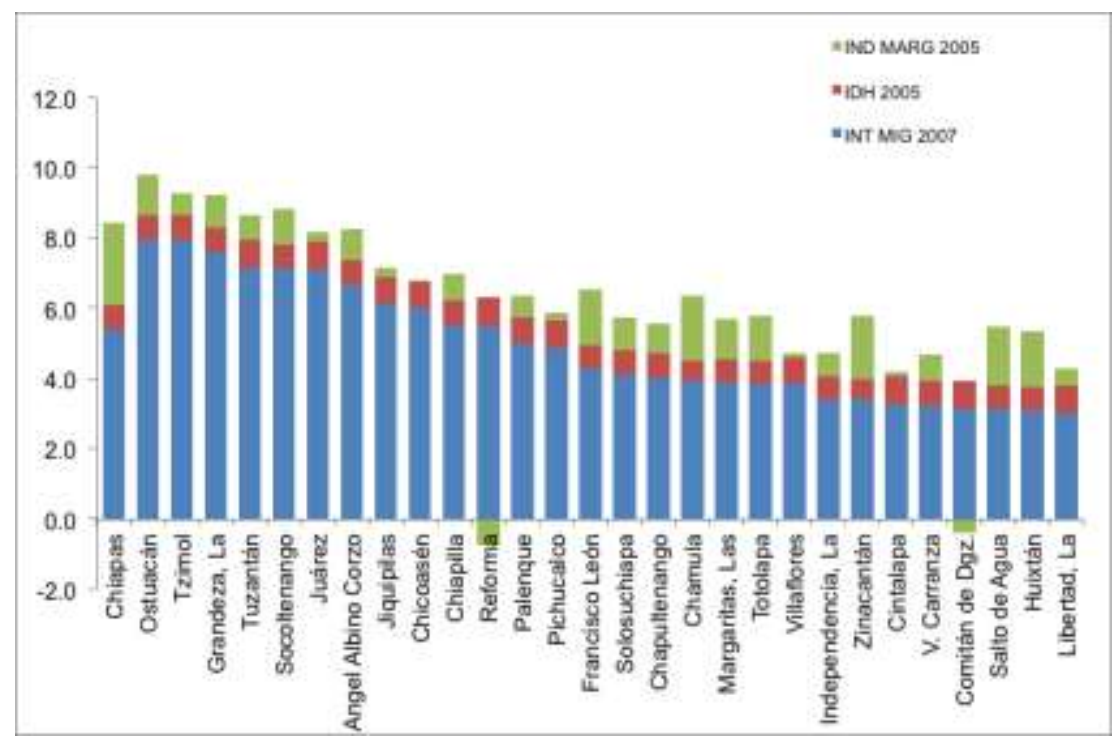

Fuente: CONAPO 2005-2007, PNUD 2007, IME 2006-2007. 
alta la conforman 28 demarcaciones municipales de distinta procedencia regional, todas con el mismo tamaño de representación de su índice de desarrollo humano pero con muy visibles variaciones en su índice de marginación.

En esta última observación se incluyen dos municipios, Reforma y Comitán, cuyo índice de marginación es negativo. Nuevamente, podemos apreciar que al inicio de la serie existen municipios de mayor tamaño en su índice de intensidad migratoria con tamaños pequeños en lo correspondiente a su índice de marginación, sin embargo, de la mitad de la serie hacia el final, encontramos que conforme desciende el índice de intensidad migratoria comienzan a saltar municipios debido a que muestran un mayor índice de marginación. Estos últimos son: Francisco León, Chamula, Zinacantán, Salto de Agua y Huixtán, tres de los cuales son eminentemente indígenas. También apreciamos que el índice de desarrollo humano se comporta visualmente homogéneo en esta categoría de municipios.

\section{MUNICIPIOS DE BAJA MIGRACIÓN}

Son 32 los municipios que conforman la categoría de baja migración, de los cuales, como se aprecia en la Figura 6, solamente dos ostentan un indicador de marginación negativo, Tuxtla Gutiérrez y San Cristóbal de Las Casas. Es evidente que en esta serie de municipios se aprecie un mayor nivel de marginación que los municipios de las dos series anteriores. En cuanto al índice de desarrollo humano, pueden apreciarse diferencias menores respecto al promedio estatal en los municipios de El Bosque, Tenejapa, Tumbalá y Chenalhó. Cabe señalar que aun cuando la serie de municipios están en la categoría de baja migración, los primeros municipios de la misma, que son los que tienen más elevada migración, exhiben mejores condiciones relativas a su índice de marginación respecto al resto de la serie. 
Figura 6. Chiapas: índice de desarrollo humano e índice de marginación, según intensidad migratoria baja 2007.

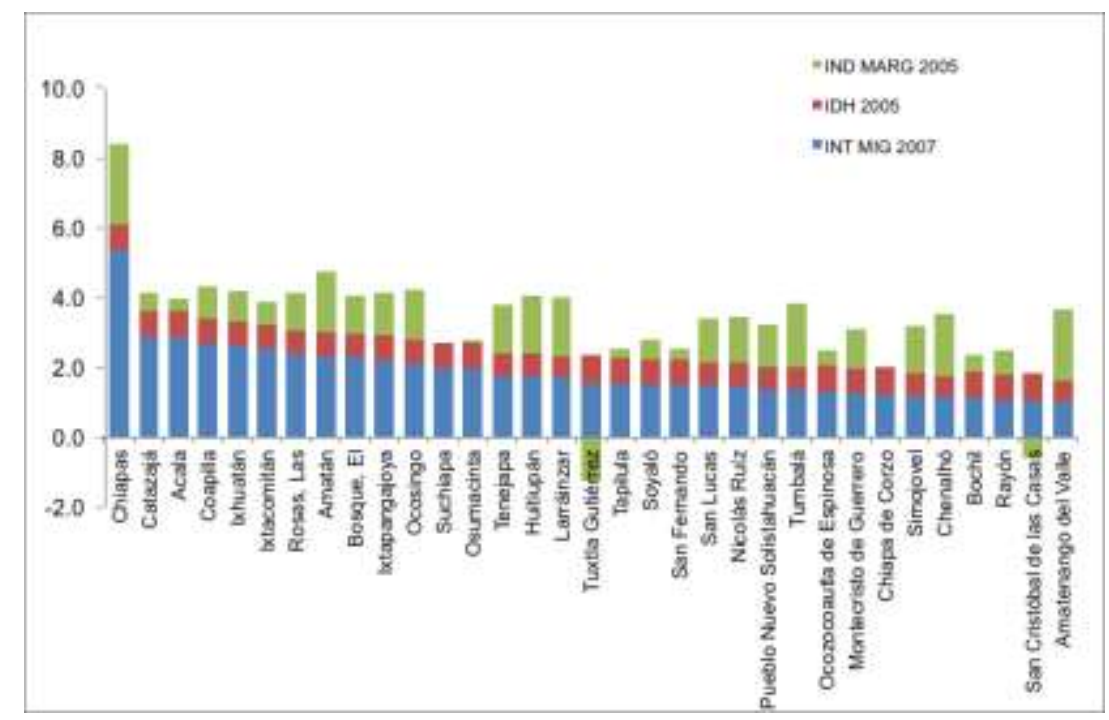

Fuente: CONAPO 2005-2007, PNUD 2007, IME 2006-2007.

\section{MUNICIPIOS DE MUY BAJA MIGRACIÓN}

La siguiente Figura 7 nos muestra de entrada la muy baja migración de todos los municipios de la serie respecto al promedio estatal y sobre todo si se compara con las dos primeras series analizadas. El número de municipios en esta categoría es de 24 municipios, de los cuales en tres la migración internacional parece estar ausente: Aldama, San Andrés Duraznal y Santiago El Pinar. Como es fácil percatarse, en esta serie se aprecia un mayor tamaño del índice de marginación respecto a la entidad y con mayor evidencia respecto a la serie correspondiente a los municipios de muy alta y alta migración (véanse Figuras 4 y 5). 
Figura 7. Chiapas: índice de desarrollo humano e índice de marginación, según intensidad migratoria muy baja 2007.

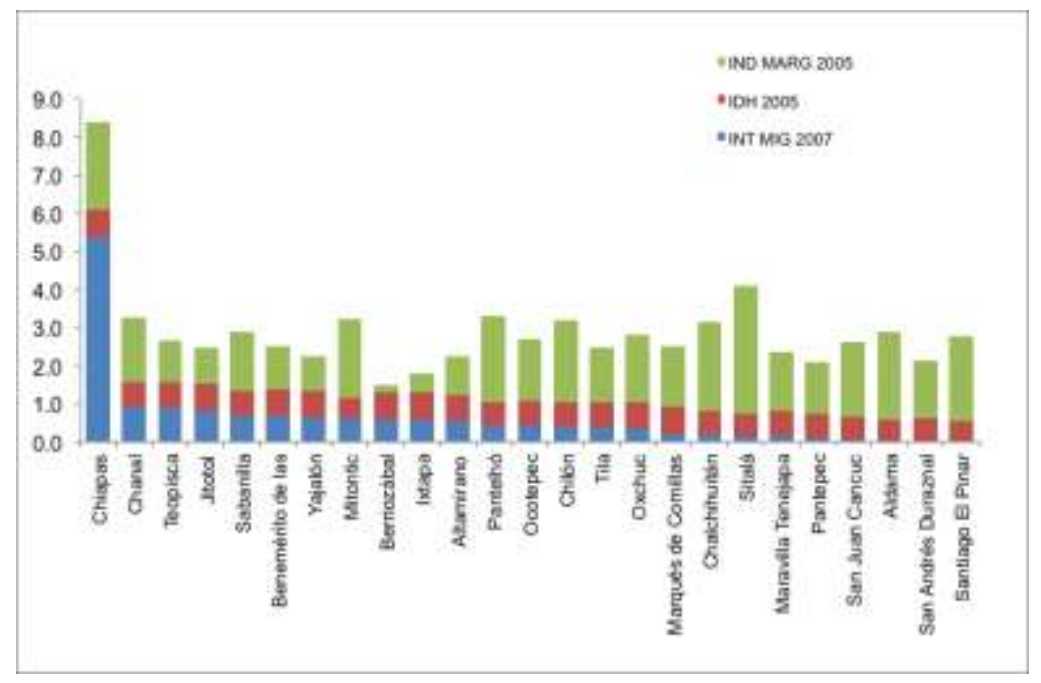

Fuente: CONAPO 2005-2007, PNUD 2007, IME 2006-2007.

En síntesis, se puede concluir en esta materia que con excepción de Tuxtla Gutiérrez existe una tendencia generalizada de que en situaciones de mayor alivio socioeconómico es mayor la proclividad a emigrar a Estados Unidos. También se constata que a mayores niveles de marginación, con excepciones de algunos municipios como el de Berriozábal, son menores los niveles de migración. También se concluye que en relación con el papel que tienen los índices de desarrollo humano y el de marginación, es este último el que resulta más sensible para determinar la asociación entre las condiciones sociales y su facilitación o no para generar procesos de migración.

Por otra parte, resulta de interés contrastar los municipios que tienen escasa o nula participación migratoria internacional — baja emigración - con el desglose de otros indicadores que componen su índice de marginación, como es el caso del tamaño de la localidad. Resulta atrayente advertir que en el indicador de población que vive en poblaciones menores a 5,000 habitantes, $70.0 \%$ del total de municipios del grupo de muy 
baja emigración tiene más de $75.0 \%$ de su población viviendo en localidades de esa categoría; $56.2 \%$ del correspondiente al grupo de baja; $64.0 \%$ del conjunto de alta, reduciéndose a $47.0 \%$ del total de municipios que componen el grupo de muy alta migración. El porcentaje promedio de población que vive en localidades menores a 5,000 habitantes en Chiapas es de 58.4.

Otro indicador sensible a la migración se refiere al porcentaje de población analfabeta. Con referencia al promedio del estado, que es de $21.3 \%$ de población que no sabe leer ni escribir, se tiene que en los municipios de muy alta emigración ostentan un promedio de 18.7\%; los de alta, 25.4\%; los de baja, 27.3\%, y por último los muy baja emigración el promedio asciende a 37. Podría argumentarse que esta tendencia está sesgada en tanto que los solicitantes de matrícula consular presumiblemente son alfabetizados, sin embargo, Chamula nuevamente sirve de ejemplo y de excepción a la regla, en el sentido de que aun cuando exhibe un porcentaje de analfabetismo que asciende a $53.1 \%$ se ha catalogado como de alta emigración con base en los registros del IME.

Estas tendencias sugieren pensar que en los municipios de escasa o nula participación migratoria la ausencia de una mayor interacción social a nivel de su entorno debido a su dispersión demográfica revelada en sus pequeños tamaños de población, más su condición de analfabetos y sus muy bajos niveles de educación, sin centros gravitacionales que integren un capital social como son los medios de comunicaciones y trasportes formales e informales, compra-venta de mercancías y servicios, además del papel todavía simbólico que las generaciones de mayores en esos contextos tienen; pudieran estar inhibiendo el acceso a la corriente migratoria hacia Estados Unidos, y en su lugar acudir colectivamente a las acostumbradas prácticas laborales de sobrevivencia que generaciones anteriores hubiesen delineado. Por último, para reafirmar las tendencias, otro indicador que de manera evidente revela una menor migración en estos municipios se constata en el índice de masculinidad que exhiben, presentándose en este grupo de muy baja emigración un valor más elevado que en el resto. En este residen 102 hombres por cada 100 mujeres, mientras 
que en el siguiente de baja es de 100; en el subsiguiente de alta es de 98; y en el de muy alta migración desciende a 96 hombres por cada 100 mujeres.

\section{CONCLUSIONES}

La migración laboral hacia Estados Unidos se ha instalado en la lógica de superación económica entre la población chiapaneca como mecanismo no solamente de obtención de recursos para el sustento diario del hogar, sino también como medio de movilidad social debido a un contexto de bajos salarios y precariedad en el trabajo. ${ }^{6}$ A las generaciones jóvenes, esta migración internacional no solo les permite resolver necesidades personales, asimismo les confiere la iniciación a nuevas prácticas culturales de reproducción social, en las que se incluyen dimensiones tan subjetivas como obtener prestigio masculino, ${ }^{7}$ que deviene en un capital social y simbólico, permitiéndoles ganar espacios personales en ambientes comunitarios. La diócesis de San Cristóbal de Las Casas documenta que «hay un contagio entre los jóvenes por salir» (Pickard 2005). La migración en este contexto se concibe entonces como una práctica social que ha venido a estructurar una serie de dimensiones asociadas a ella, tales como las redes sociales intra y extra comunitarias, la conformación del coyotaje local, regional y trasnacional, más la generación de un ambiente colectivo en los espacios de origen, incluyendo los visos de organización en los de destino, elementos todos que dinamizan de nueva cuenta nuevos flujos de migración.

Al exhibir la entidad situaciones de estancamiento económico, que no permiten visualizar en el mediano plazo capacidades para incorporar a la creciente mano de obra en condiciones salariales satisfactorias, la migración será el medio para refuncionalizar las economías familiares del campo y la ciudad. De ahí que la base para mantener la emigración internacional a Estados Unidos descanse, por una parte, en las condiciones económicas que acusa la entidad $\mathrm{y}$, por otra, en el tejido que las redes sociales vayan desplegando cuyos alcances lleguen a constituir la verdadera base de sobrevivencia para cantidades indeterminadas de hogares chiapanecos. Esta previsión se basa en la experiencia de las entidades tradicionales de migración, en las que aun cuando tienen mayores 
posibilidades de desarrollo, mediante sus redes mantienen una articulación sólida con el mercado laboral de Estados Unidos. Véase los casos de Jalisco, Zacatecas, Michoacán, Guanajuato, que siendo entidades de mayor desarrollo que la chiapaneca sus flujos de migración todavía persisten.

Las nuevas generaciones de migrantes presentan un proceso de ruptura generacional respecto a los ámbitos laborales, en tanto que han dejado atrás los tradicionales destinos migratorios cuyos padres durante décadas asistieron para abrir nuevos destinos laborales dentro y fuera del país. Lo anterior se relaciona con la configuración de nuevas expectativas de vida y de horizontes de trabajo, que implican nuevas estrategias para ganarse la vida.

La búsqueda de alternativas laborales vía emigración internacional ha sido generalizada por parte de casi todas las regiones y municipios de Chiapas, trátese de campesinos, indígenas y, sin duda, de mestizos empobrecidos del campo y la ciudad. Como pudo apreciarse, si bien es cierto que la entidad acusa los mayores índices de marginación y los más bajos de desarrollo humano, son los municipios en condiciones sociales relativamente mejores en donde se generan los mayores flujos de migración. Sin duda que esto se debe a que los costos de una migración de larga distancia con grandes dificultades en el cruce se ubican como los determinantes para diferenciar de quiénes pueden o no pagar el cobro económico.

En complemento de lo anterior, cabe destacar que la migración ha sido un proceso que ha conjuntado una estrategia común: a diferencia del pasado, cuando las alternativas eran segregacionistas y se diferenciaban por la adscripción étnica y por el lugar de residencia —rural-urbano-, e inserciones laborales en destino, ahora la migración como estrategia de vida ha borrado casi completamente esas distinciones laborales para dar cuenta a una integración de estrategias de sobrevivencia del conjunto de la población chiapaneca, apuntando a un destino laboral común.

El conjunto de lo antes expuesto nos conduce a advertir que la migración chiapaneca ha 
entrado en una fase acelerada de incursión laboral en EUA, propiciada no solamente por las determinantes sociales y económicas acaecidas en los últimos decenios en la entidad, sino también por las nacientes redes sociales que han venido surgiendo en la multitud de localidades de origen, incluyendo la emergencia de facilitadores que inducen a la población joven a insertarse en el proceso migratorio.

Respecto a las redes, cabe señalar que si bien es cierto paulatinamente han venido cobrando importancia, no han logrado estar a la altura de las circunstancias que impone el cruce y el despliegue logístico en la Unión Americana en términos de instalación, localización de oportunidades de empleo y, sobre todo, respecto al tiempo de respuesta ante las eventualidades de emergencia registradas a la llegada del migrante, riesgos de la calle y del trabajo, y de comunicaciones con los lugares de origen. De igual forma, tampoco han llegado al nivel que exige imprimir nuevos senderos sociales y económicos a la población en los lugares de origen mediante la potenciación de la migración vía planteamientos de desarrollo y de participación social de la comunidad. El tránsito entre las exiguas ganancias obtenidas de la migración que hasta hoy ha imperado en el grueso de la población migrante se circunscribe al ámbito de la unidad familiar, sin mayores consecuencias en el ámbito comunitario. Más aún, si los dividendos ocurren a nivel familiar, o en el peor de los casos individualmente, se estará en una situación de una mayor diferenciación social entre los que migran y los que no lo hacen por carecer del recurso.

\section{FUENTES DE CONSULTA}

Banco de México, varios años. Sistema de Información Económica (SIE), Indicadores Económicos, en www.banxico.org.mx [consulta: 22 de abril de 2012].

Benjamin, Thomas, 1995, Chiapas: tierra rica, pueblo pobre. Historia política y social, Grijalbo, México.

Consejo Estatal de Población del estado de Chiapas, 2007, «Panorama reciente de la 
migración en Chiapas», en Graciela Freyermuth, Sergio Meneses y Germán Martínez (coords.), El señuelo del norte. Migración indígena contemporánea, Coespo, Fondo de Población de Naciones Unidas, Asesoría, Capacitación y Asistencia en Salud, A. C., San Cristóbal de Las Casas, Chiapas.

Consejo Nacional de Población, 2002, «Índice de intensidad migratoria por entidad federativa», Conapo, México.

Consejo Nacional de Población, 2005, «Migración México-Estados Unidos. Panorama regional y estatal», Conapo, México.

Consejo Nacional de Población, Instituto Nacional de Geografía y Estadística, Secretaría de Salud, Instituto Nacional de Salud Pública,2006, «Encuesta Nacional de la Dinámica Demográfica 2006», Conapo, Inegi, SS, INSP, México.

Encuesta de Migración de la Frontera Norte, 2006, Bases de datos de la Encuesta sobre Migración en la Frontera Norte de México, 1995, 1999-2006, EMIF, COLEF, Conapo, STPS, SRE, INM, México.

García de León, Antonio, 1985, Resistencia y Utopía. Memorial de agravios y crónica de revueltas y profecías acaecidas en la provincia de Chiapas durante los últimos 500 Años de su historia, 2 tomos, Ediciones ERA, México.

García de León, Antonio, 2002, Fronteras interiores. Chiapas: una modernidad particular, Editorial Océano, México.

Instituto de los Mexicanos en el Exterior, 2007, 2010, Registros Administrativos. Instituto de los Mexicanos en el Exterior, IME, Dirección General de Protección y Asuntos Consulares, Secretaría de Relaciones Exteriores, México. 
Instituto Nacional de Estadística y Geografía, 1970, 1990, 2000, X, XI, XII Censo General de Población y Vivienda, Inegi, México.

Instituto Nacional de Geografía y Estadística, 2004, Encuesta Nacional de Empleo, módulo de migración, 2002, Inegi, México.

Instituto Nacional de Estadística y Geografía, 2005, II Conteo de Población, Inegi, México.

Instituto Nacional de Estadística y Geografía, 2009, Censo Agropecuario 2007, VIII Censo Agrícola, Ganadero y Forestal, Inegi, México.

López Arévalo, Jorge y Bruno Sovilla, 2009, «La globalización por la vía neoliberal y la emigración en Chiapas», en Jorge López Arévalo et al., Globalización, Migración y Economía Chiapaneca, Universidad Autónoma de Chiapas, México.

Martínez Velasco, Germán, 1994, Plantaciones, trabajo guatemalteco y política migratoria en la Frontera Sur, Instituto Chiapaneco de Cultura, Gobierno del estado de Chiapas, México.

Martínez Velasco, Germán, 1999a, «Globalización y subdesarrollo local: diferenciación social y migración en Chiapas», Papeles de Población, v. 5, n. 22, UAEM, México.

Martínez Velasco, Germán, 1999b, «Por qué quedarse en Chiapas. Un análisis de la migración intra-estatal», Tesis de doctorado, El Colegio de la Frontera Norte, México.

Nájera Aguirre, Jéssica y Jorge López Arévalo, 2009, «Migración de chiapanecos a los Estados Unidos de América, una visión desde la Encuesta sobre Migración en la Frontera Norte de México-EMIF NORTE», I Congreso Internacional sobre Pobreza, Migración y 
Desarrollo, 22-24 de abril, San Cristóbal de las Casas, Chiapas, México.

Pickard, Miguel, 2005, Entre fuegos cruzados: Los migrantes mesoamericanos en su travesía hacia el norte, Programa de las Américas, Interhemispheric Resource Center, Nuevo México.

Programa de Naciones Unidas para el Desarrollo, 2007, Informe sobre Desarrollo Humano. México, 2006-2007, PNUD, México.

Pozas A., Ricardo, 1952, «El trabajo en las plantaciones de Café y el cambio sociocultural del indio», Revista Mexicana de Estudios Antropológicos, v. 13, n. 1, México.

Rus Diana y Jan Rus, 2008, «La migración de trabajadores indígenas de Los Altos de Chiapas a Estados Unidos, 2001-2005: el caso de San Juan Chamula», en Daniel Villafuerte y Ma. del Carmen García Aguilar (coords.), Migraciones en el Sur de México y Centroamérica, UNICACH, Miguel Ángel Porrúa, México.

Secretaría de Desarrollo y Participación Social, 2010, Programas sociales convenidos, Ramo Administrativo 20, Tuxtla Gutiérrez, Chiapas.

Secretaría del Trabajo y Previsión Social, 2007, «Los Braceros», en Durand, Jorge (comp.), Braceros, las miradas mexicanas y estadounidense, Antología (1945-1964), Senado de la República, Universidad de Zacatecas, Miguel Ángel Porrúa, México.

Secretaría para el Desarrollo de la Frontera Sur, 2010, Boletines, registros y documentos internos, Gobierno del estado de Chiapas, Tapachula, Chiapas.

Spenser, Daniela, 1988, El Partido Socialista chiapaneco, Ediciones de la Casa Chata, Ciesas, México. 
Wasserstrom, W. Robert, 1980, Ingreso y trabajo rural en los Altos de Chiapas: El caso de San Juan Chamula, Centro de Investigaciones Ecológicas del Sureste, México.

\section{NOTAS}

${ }^{1}$ Además de lo señalado, debe tomarse en cuenta una serie de peripecias para la obtención de las tarjetas de trabajo que servían para ser considerado como aspirante a bracero, situación que propició una serie de corruptelas entre las autoridades de la Secretaria del Trabajo y Previsión Social.

${ }^{2}$ Para un recuento de la historia de desigualdad rural en Chiapas, véase García de León Antonio, Resistencia y Utopía, frontera interiores: Chiapas una modernidad particular, Océano, 2002; Benjamín T., 1995, Chiapas, Tierra rica, pueblo pobre. Historia política y social.

${ }^{3}$ Testimonios levantados en trabajo de campo, del proyecto "La cafeticultura en El Soconusco". Centro de Investigaciones Ecológicas del Sureste. 1990.

${ }^{4}$ Cabe aclarar que las cifras que arroja el IME son bastante valiosas para identificar la distribución de chiapanecos en Estados Unidos, así como de otras características sociodemográficas, principalmente en lo concierne al conocimiento de los lugares de origen a nivel municipal.

${ }^{5}$ www. sedesol.gob.mx/transparencia/transparencia_iniciativa_3 x 1.htm

${ }^{6}$ Se sostiene la tesis que no es la falta de empleo lo que induce a emigrar sino los bajos salarios que privan, ya que de acuerdo con EMIF, poco más de $80.0 \%$ de los chiapanecos entrevistados en la frontera norte declararon tener un empleo previo en la entidad. Promedio arriba del nacional que es de 65.0\% (EMIF-NORTE, 2007).

${ }^{7}$ En los estudios antropológicos y de género, se reitera la noción de prestigio entre los hombres adquiridos mediante la migración a Estados Unidos.

Fecha de recepción: 27 de septiembre de 2012.

Fecha de aceptación: 15 de octubre de 2012. 